Stability of a Sloping Interface in a Rotating Two-Fluid System

\author{
By \\ Desiraja B. Rao and T.J. Simons
}

Department of Atmospheric Science

Colorado State University

Fort Collins, Colorado

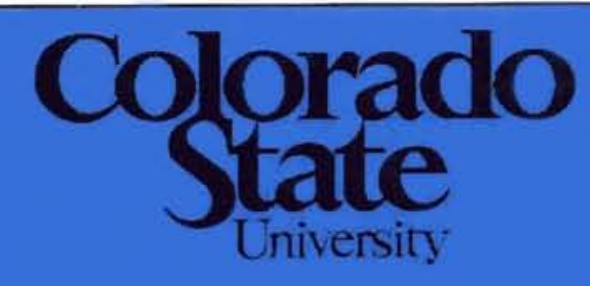

Department of
Atmospheric Science

Paper No. 151 
STABILITY OF A SLOPING INTERFACE

IN A ROTATING TWO-FLUID SYSTEM

by

Desiraju B. Rao

and

T. J. Simons

This research was supported by NSF GA-11637

U.S.A.F. F 19628-68-C-0104

Department of Atmospheric Science Colorado State University

Fort Collins, Colorado

November 1969

Atmospheric Science Paper No. 151 
Table of Contents

Page

ABSTRACT

1. Introduction 1

2. Dynamical Equations 5

3. Method of Solution 7

4. Results 10

5. Other Cases of Mixed Mode Instability 23

6. Summary and Conclusions 29

$\begin{array}{ll}\text { ACKNOWLEDGEMENTS } & 31\end{array}$

$\begin{array}{ll}\text { REFERENCES } & 32\end{array}$ 
Abstract

When two incompressible homogeneous fluids in relative motion are superimposed in a gravitationally stable configuration and are subjected to rotation about a vertical axis, the interface and the free surface slope away from the horizontal. Such a system possesses rotational modes of oscillation in addition to the external and internal gravitational modes. The dynamic stability of small amplitude motions superimposed on this system is studied. For unstable modes, the values for the phase speed and the growth rates are presented as a function of wavelength of the perturbations and shear of the basic flow. In particular, it was shown that the instability occurs in this model as a result of an interaction between an internal gravitational mode and a rotational mode. 
I. Introduction

We are concerned here with the dynamic stability of the equilibrium configuration shown in Figure 1 with respect to small amplitude perturbations. This configuration consists of two incompressible homogeneous fluids of densities $\rho_{1}$ and $\rho_{2}$ superimposed one on top of another with the heavier fluid $\left(\rho_{1}\right)$ at the bottom to insure gravitationall stability of the system. These fluids are laterally bounded by rigid walls at $y=0$ and $y=W$ and each fluid is moving in the positive $\mathrm{x}$-direction with a constant translational velocity $\overline{\mathrm{u}}_{1}$ and $\overline{\mathrm{u}}_{2}$ $\left(\bar{u}_{1} \neq \bar{u}_{2}\right.$ in general). In the absence of rotation, the fluids will have uniform depths $\left(\bar{D}_{1}\right.$ and $\vec{D}_{2}$ as shown by the dotted lines in Figure l). In this case, the system is only capable of exhibiting gravitational (external and internal) modes of oscillation and any dynamic instability that is realized is purely a manifestation of the Kelvin-Helmholtz instability. As is well known, this instability is usually restricted to rather small wavelengths of the perturbations. If, however, we now assume that the system is rotating about a vertical axis with angular speed $\Omega$, the depths of the fluids can no longer be uniform. The translational velocities $\vec{u}_{1}$ and $\vec{u}_{2}$ bring into play the Coriolis forces which would then require balancing pressure gradients. These are generated by the interface and free surface sloping away from the horizontal as shown in Figure 1. Under these conditions, the system exhibits an additional mode of oscillation--the rotational mode which is normally referred to as the Rossby mode in meteorology--in addition to the gravity modes. The features of these rotational modes normally resemble the characteristics of meteorologically important 


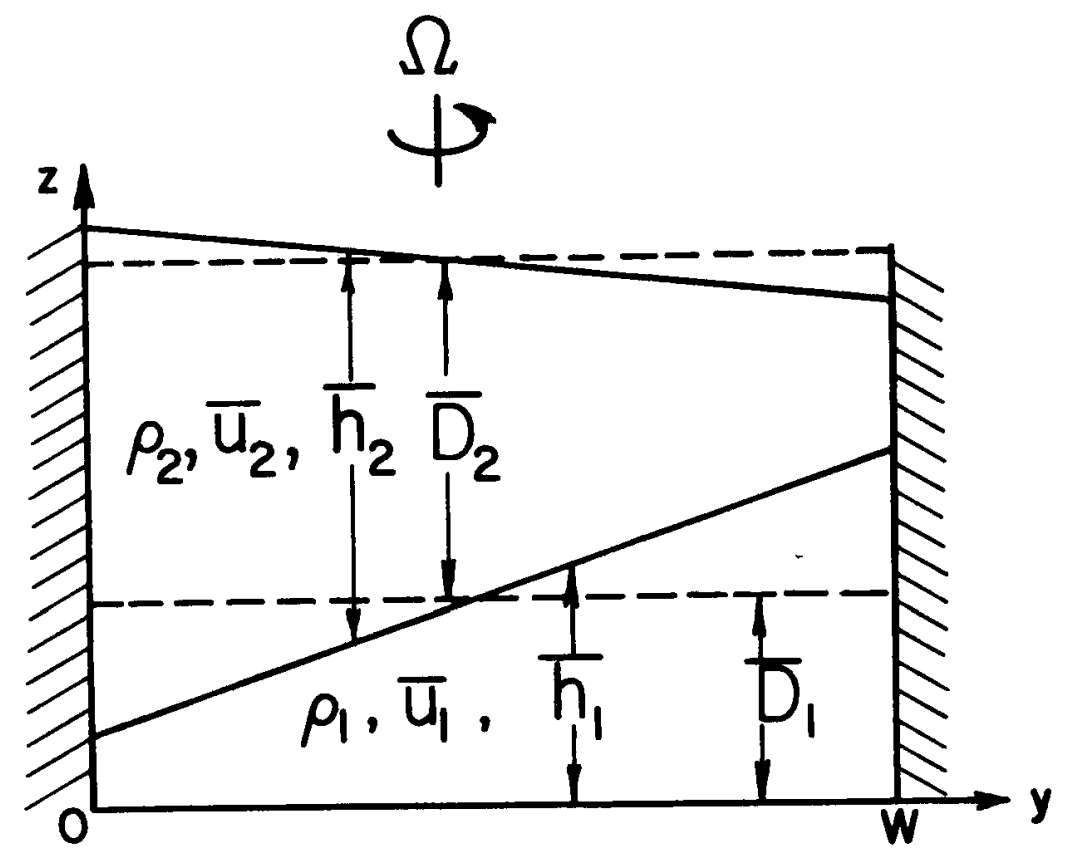

Figure 1. Equilibrium configuration of the physical model. 
wave motions that are observed on synoptic weather maps. Our primary concern here is to investigate the stability of the basic state which possesses both the gravitational and rotational modes.

The study of the above stability problem has an approximate applicability to the problem of frontal instability in meteorology. The Bergen school of Norway has demonstrated that instability associated with narrow transition zones between warm and cold air masses is a possible factor in determining the initial growth of a perturbation in the middle latitudes. In an idealized situation, one can represent each of these air masses by a homogeneous incompressible fluid and the transition zone by a surface of zero-order discontinuity -called the front-with respect to density and tangential velocities. The dominant characteristics of the frontal model are that the frontal surface is inclined at an angle to the horizontal and intersects the ground at some point. These two features together complicate the necessary mathematical analysis to determine the stability characteristics of the model. One of the earliest attempts at a mathematical analysis of this model is that of Solberg (1928). His model consisted of a rigid top and bottom both of which are inclined at the same angle to the horizontal as the frontal surface. In spite of this simplicity, Solberg's results showed that it is indeed possible to have unstable waves whose wavelengths are approximately in the range of incipient cyclones when the frontal surface is no longer horizontal. It may be recalled that without the sloping front, the only instability the twofluid system can give is the Kelvin-Helmholtz instability, which is usually confined to wavelengths much smaller than the scale of the incipient cyclones for reasonable values of shear $\left(\bar{u}_{2}-\bar{u}_{l}\right)$. 
Subsequent to Solberg, the mathematical analysis of the frontal problem was done by Kotschin (1932) and Eliasen (1960) with rigid top models in which the frontal surface intersects the ground. Recently Orlanski (1968) made a more elaborate analysis of the Kotschin model.

Most of the traditional stability analyses in meteorology are made for the long planetary waves for which the so-called quasi-geostrophic approximation is introduced. This approximation, first introduced by Charney (1947), effectively filters out the gravity waves leaving only the rotational waves in the system. Use of this approximation greatly simplifies the necessary mathematical analysis. However, when one is concerned with the stability of perturbations of a smaller scale--as those on a frontal surface--this approximation can no longer be justified. Consequently, one has to deal with a non-geostrophic system containing both gravitational and rotational modes. There have been some studies of this ageostrophic stability problem (Arnason 1963, Derome and Wiin-Nielsen 1966, Stone 1966, Sela and Jacobs 1968) but the precise nature of the stability character and the interaction between the gravitational and rotational modes, if any, is not properly brought out. In the previously mentioned studies on frontal stability, the analysis of the problem was quite complicated in view of the frontal intersection with the ground and the role of the ageostrophic effects introduced by the presence of the gravity waves is not clearly brought out either. It is with a view to investigate this aspect while trying to keep the necessary analysis simple, that we selected the model described earlier. Since the characteristic feature of the frontal theory is the inclination of the front, rather than its intersection with the ground, the results obtained here may still. be applicable in an 
approximate sense to a frontal situation.

\section{Dynamical Equations}

The physical model is described in the preceding section. As mentioned there, we are dealing with motions whose scales are much smaller than the radius of the earth. Consequently, these motions may be described using a Cartesian $x, y-p l a n e$ with a constant Coriolis parameter $f=2 \Omega \sin \phi, \Omega$ being the angular speed of rotation and $\phi$ is the goegraphical latitude. We further assume that the scale of the motions is such that the hydrostatic-or the long wave-approximation is valid. Then the motion in each layer can be described by the shallow water equations:

$$
\begin{aligned}
& \frac{d V_{1}}{d t}-f\left[V_{1}\right]=-g\left(\varepsilon \nabla h_{2}+\nabla h_{1}\right) \\
& \frac{d h_{1}}{d t}+h_{1} \nabla \cdot V_{1}=0 \\
& \frac{d V_{2}}{d t}-f\left[V_{2}\right]=-g\left(\nabla h_{2}+\nabla h_{1}\right) \\
& \frac{d h_{2}}{d t}+h_{2} \nabla \cdot I V_{2}=0
\end{aligned}
$$

In these equations, the subscript 1 refers to the quantities in the lower layer and subscript 2 to those in the upper layer. $V=(u, v)$ is the horizontal velocity vector, $h$ is the depth of a layer. The vector [V] denotes a rotation of IV through ninety degrees in the negative sense of the $x, y-p l a n e . ~ \varepsilon \equiv \rho_{2} / \rho_{1}$ is the density ratio and for a gravitationally stable configuration $0<\varepsilon<1$. The system of equations (2.1) to (2.4) has an exact solution corresponding to the basic state 


$$
\begin{array}{ll}
\overline{\mathrm{V}}_{1}=\left(\overline{\mathrm{u}}_{1}, 0\right) & \overline{\mathrm{V}}_{2}=\left(\overline{\mathrm{u}}_{2}, 0\right) \\
\frac{\mathrm{d} \overline{\mathrm{h}}_{1}}{\mathrm{dy}}=\bar{\alpha}_{1} \mathrm{f} & \frac{\mathrm{d} \overline{\mathrm{h}}_{2}}{\mathrm{dy}}=\bar{\alpha}_{2} \mathrm{f}
\end{array}
$$

where we have defined

$$
\bar{\alpha}_{1} \equiv \frac{\varepsilon \bar{u}_{2}-\bar{u}_{1}}{g(1-\varepsilon)} \quad \bar{\alpha}_{2} \equiv \frac{\bar{u}_{1}-\bar{u}_{2}}{g(1-\varepsilon)}
$$

We now assume that the translational velocities $\bar{u}_{1}$ and $\bar{u}_{2}$ in the basic state are constant $\left(\bar{u}_{1} \neq \bar{u}_{2}\right)$ and that the domain of interest is bounded by rigid boundaries at $y=0$ and $y=W$. The depths of the lower and upper fluid layers are then given by (for $\mathrm{y}>0$ ):

$$
\overline{\mathrm{h}}_{1}=\overline{\mathrm{D}}_{1}+\bar{\alpha}_{1} \mathrm{f}\left(\mathrm{y}-\frac{\mathrm{W}}{2}\right) \quad \overline{\mathrm{h}}_{2}=\overline{\mathrm{D}}_{2}+\bar{\alpha}_{2} \mathrm{f}\left(\mathrm{y}-\frac{\mathrm{W}}{2}\right) \quad(2.7)
$$

where $\bar{D}_{1}$ and $\bar{D}_{2}$ are the layer depths at $y=W / 2$. They are also the uniform depths that the fluid layers will have in the absense of rotation.

The dynamical equations (2.1-4) are now linearized about the state of equilibrium expressed through equations $(2.5,2.7)$ for the examination of the stability problem. The linearized equations are

$$
\begin{aligned}
& L_{j} u_{j}-f v_{j}+g\left(\frac{\partial h_{1}}{\partial x}+\gamma_{j} \frac{\partial h_{2}}{\partial x}\right)=0 \\
& L_{j} v_{j}+f u_{j}+g\left(\frac{\partial h_{1}}{\partial y}+\gamma_{j} \frac{\partial h_{2}}{\partial y}\right)=0 \\
& L_{j} h_{j}+\bar{\alpha}_{j} f v_{j}+\bar{h}_{j}\left(\frac{\partial u_{j}}{\partial x}+\frac{\partial v_{j}}{\partial y}=0\right.
\end{aligned}
$$

where $j=1$ or 2 and $u_{j}, v_{j}, h_{j}$ are perturbation quantities.

We have defined

$$
\begin{aligned}
& \gamma_{1} \equiv \varepsilon \text { and } \gamma_{2} \equiv 1 \\
& L_{j} \equiv \frac{\partial}{\partial t}+\bar{u}_{j} \frac{\partial}{\partial x}
\end{aligned}
$$

The equations (2.8-10) represent a coupled set of six homogeneous 
equations in as many unknowns. The necessary boundary conditions to be adjoined in order to complete the specification of the problem are:

$$
\begin{aligned}
& u_{j}, v_{j}, h_{j} \text { are periodic in } x \\
& \text { and } v_{j}=0 \text { at } y=0 \text { and } w
\end{aligned}
$$

In the next section we outline the method of solution for the boundary value problem (2.8-12).

\section{Method of Solution:}

The coefficients in the dynamical equations (2.7-10) are constants in $\mathrm{x}$ so that a solution of the form

$$
\begin{gathered}
u_{j}=U_{j}(y, t) e^{i k x}, v_{j}=v_{j}(y, t) e^{i k x} \\
j=1,2 .
\end{gathered}
$$

automatically satisfies the equations and the boundary condition in $\mathrm{x}$. Substitution of this into the equations yields

$$
\begin{aligned}
& \frac{\partial U_{j}}{\partial t}+i k \bar{u}_{j} U_{j}-f V_{j}+i k g\left(H_{1}+\gamma_{j} H_{2}\right)=0 \\
& \frac{\partial V_{j}}{\partial t}+i k \bar{u}_{j} V_{j}+f U_{j}+g\left(\frac{\partial H_{1}}{\partial y}+\gamma_{j} \frac{\partial H_{2}}{\partial y}\right)=0 \\
& \frac{\partial H_{j}}{\partial t}+i k \bar{u}_{j} H_{j}+\bar{\alpha}_{j} f V_{j}+\bar{h}_{j}\left(i k U_{j}+\frac{\partial V_{j}}{\partial y}\right)=0
\end{aligned}
$$

with boundary conditions $v_{j}=0$ at $y=0$ and $w$

This system of equations has been solved by spectral and by finite difference methods. Considering first the spectral solution, we seek solutions of the form

$$
v_{j}(t, y)=\sum_{\ell=0}^{\infty} v_{j}^{\ell}(t) \sin \frac{\ell \pi y}{W}
$$

in order to satisfy the lateral boundary conditions. Since now $v_{j}$ is an odd function of $y$, it follows from the equations that $U_{j}$ and $H_{j}$ are 
even functions of $y$, thus

$$
\begin{aligned}
& U_{j}(t, y)=\sum_{l=0}^{\infty} V_{j}^{\ell}(t) \cos \frac{l \pi y}{W} \\
& H_{j}(t, y)=\sum_{l=0}^{\infty} H_{j}^{\ell}(t) \cos \frac{l \pi y}{W}
\end{aligned}
$$

and further $f$ is an odd function and $\bar{h}_{j}$ is an even function of $y$ to satisfy the appropriate parities of all quantities in a mathematical sense. Again on a mathematical plane, if all integrations are performed over an interval $-\mathrm{W}<\mathrm{y}<\mathrm{W}$, then sines and cosines are orthogonal and all odd functions integrate out.

Substituting the expansions $(3.6,3.7)$ into the dynamical equations $(3.2,3.3,3.4)$, multiplying the $U_{f}, H_{j}$ - equations by $\cos \frac{n \pi y}{W}$ and the $v_{j}$ - equation by $\sin \frac{n \pi y}{w}$, and integrating over the interval $0<y<w$, we obtain the spectral equations

$$
\begin{aligned}
& \frac{d U_{j}^{n}}{d t}+i k \bar{u}_{j} U_{j}^{n}-\sum_{\ell=0}^{\infty} \delta_{n} f^{\ell n} v_{j}^{\ell}+i k g\left(H_{l}^{n}+\gamma_{j} H_{2}^{n}\right)=0 \\
& \frac{d v_{j}^{n}}{d t}+i k \bar{u}_{j} v_{j}^{n}+\sum_{\ell=0}^{\infty} f^{n \ell} U_{j}^{\ell}-\frac{n \pi g}{W}\left(H_{l}^{n}+\gamma_{j} H_{2}^{n}\right)=0 \\
& \frac{d H_{j}^{n}}{d t}+i k \bar{u}_{j} H_{j}^{n}+\sum_{\ell=0}^{\infty} \delta_{n}\left[\bar{\alpha}_{j} f^{\ell n_{j}^{\ell}}+\bar{h}_{j}^{\ell n}\left(i k U_{j}^{\ell}+\frac{\ell \pi}{W} v_{j}^{\ell}\right)\right]=0
\end{aligned}
$$

where $n=0,1,2, \ldots \ldots, \delta_{n}=1$ for $n \neq 0$ and $\delta_{n}=\frac{1}{2}$ for $n=0$, and the coefficients are defined as follows:

$$
\begin{aligned}
& f^{\ell n} \equiv \frac{2}{W} \int_{0}^{W} f \sin \frac{\ell \pi y}{W} \cos \frac{n \pi y}{W} d y \\
& \bar{h}_{j} \ell n=\frac{2}{W} \int_{0} W \bar{h}_{j} \cos \frac{\ell \pi y}{W} \cos \frac{n \pi y}{W} d y
\end{aligned}
$$

which can be computed once $f$ and $\bar{h}_{j}$ have been specified as functions of $\mathrm{y}$.

For computational purposes the series must be truncated, say at $\mathrm{n}=\mathrm{N}$. We have then a system of $6(\mathrm{~N}+1)$ prediction equations, but 
since (3.9) can be discarded for $n=0$, we have only $6 N+4$ coupled equations. The system can be cast into the form

$$
\frac{d \vec{a}}{d t}+i k / A \vec{a}=0
$$

where $\vec{a}$ is the column vector consisting of the $6 \mathrm{~N}+4$ time-dependent variables and $A$ is a matrix of order $(6 N+4) \times(6 N+4)$ with timeindependent elements. The solution of (3.12) is of the form

$$
\vec{a} \sim e^{-i k c t}
$$

Substitution of (3.13) into (3.12) yields the equation:

$$
(/ A-c I) \vec{a}=0
$$

which shows that the c's are the characteristic values of the matrix A. Combining this time-dependent part of the solution with the $x-$ dependent part given by (3.1) we see that the perturbation quantities $u_{j}, v_{j}, h_{j}$ are proportional to $\exp [i k(x-c t)]$. Thus a positive real value for $c$ represents waves progressing in the positive $x$-direction. If $c$ is complex then a positive imaginary part will give an exponential growth in time for the perturbations, which are then considered unstable. In the present problem complex roots occur in conjugate pairs, thus any growing root is accompanied by a decaying root. However in the Fourier integral representation of an arbitrary disturbance, this decaying wave would not make any significant contribution.

For: an independent test of the spectral solution we have also obtained the solutions to the system (3.2) through (3.5) by finite difference methods. The boundary conditions (3.5) suggest the use of a staggered grid system. The channel width $W$ is subdivided into $(N+1)$ strips, the variables $U_{j}$ and $H_{j}$ are defined at the centers of 
the strips, the variables $v_{j}$ are defined at the boundaries (where they are zero) and between the strips. Derivatives with respect to $y$ are replaced by central differences. Again we obtain a system of $6 \mathrm{~N}+4$ variables which are only time-dependent and the system can be written in the form (3.12).

The stability calculations discussed in the following section have been computed by both methods. The results were essentially the same, but the spectral method showed somewhat better convergence and therefore only the spectral results are presented in the following. Table 1 shows a typical convergence pattern for both methods. The occurrence of the two pairs of complex roots will be explained in the following section.

4. Resu1ts

In determining the characteristic values $c$ of equation (3.14), it is necessary to prescribe the values of the following parameters: $\bar{D}_{1}$, $\overline{\mathrm{D}}_{2}, \varepsilon, \overline{\mathrm{u}}_{1}, \overline{\mathrm{u}}_{2}, \mathrm{~W}, \mathrm{k}$. In most of the cases, the numerical values assigned to the various parameters are:

$$
\begin{aligned}
& \overline{\mathrm{u}}_{1}=10 \mathrm{~m} / \mathrm{sec} \\
& \varepsilon=0.98 \\
& W=500 \mathrm{~km} \\
& \bar{D}_{1}=\bar{D}_{2}=5 \mathrm{~km}
\end{aligned}
$$

Then the values of the wave number $k$ and speed of the upper layer $\bar{u}_{2}$ were varied while determining the characteristic values $c$. The numerical values for complex c's are presented in Table 2 for a truncation of the spectral series at $N=9$ in terms of the phase speed $c_{r}$ and the $e-$ folding time $1 / \mathrm{kc}_{i}$ or the perturbations. This then gives a matrix of 
Table 1. Convergence test for complex eigen values: Wave speeds $c_{r}$ and e-folding times $1 / \mathrm{kc}_{i}$ as a function of truncation, $N$. $k$ = wave number, $F D=$ finite difference method, $S P=$ spectral solution. In this table $\bar{u}_{]}=10 \mathrm{~m} \mathrm{sec}, \bar{u}_{2}=40 \mathrm{~m} \mathrm{sec}, \varepsilon=.98, W=500 \mathrm{~km}, \bar{D}_{1}=\bar{D}_{2}=5 \mathrm{~km}$.

$$
\mathrm{kW}=1 \quad \mathrm{~kW}=2
$$

\begin{tabular}{|c|c|c|c|c|}
\hline & FI) & $\mathrm{SP}$ & FD & SP \\
\hline$\underline{N}$ & $c_{Y} \quad 1 / k c_{i}$ & & & \\
\hline 1 & $12.92 \pm 2.493$ & $14.14 \pm 1.878$ & $12.86 \pm 1.529$ & $14.01+1.124$ \\
\hline & $37.09 \pm 2.530$ & $35.86 \pm 1.905$ & $37.17 \pm 1.526$ & $36.02 \pm 1.124$ \\
\hline 3 & $14.34 \pm 2.018$ & $14.73 \pm 1.928$ & $14.33 \pm 1.264$ & $14.72 \pm 1.206$ \\
\hline & $35.69 \pm 2.060$ & $35.30 \pm 1.973$ & $35.73 \pm 1.260$ & $35.35 \pm 1.205$ \\
\hline 5 & $14.62 \pm 1.964$ & $14.81 \pm 1.927$ & $14.63 \pm 1.237$ & $14.82 \pm 1.215$ \\
\hline & $35.41 \pm 2.008$ & $35.22 \pm 1.973$ & $35.44 \pm 1.233$ & $35.25 \pm 1.212$ \\
\hline 7 & $14.72 \pm 1.946$ & $14.84 \pm 1.926$ & $14.73 \pm 1.228$ & $14.85 \pm 1.217$ \\
\hline & $35.31 \pm 1.992$ & $35.20 \pm 1.973$ & $35.34 \pm 1.225$ & $35.23 \pm 1.214$ \\
\hline 9 & $14.77 \pm 1.938$ & $14.85 \pm 1.926$ & $14.78 \pm 1.225$ & $14.86 \pm 1.218$ \\
\hline & $35.26 \pm 1.985$ & $35.19 \pm 1.973$ & $35.29 \pm 1.221$ & $35.21 \pm 1.215$ \\
\hline
\end{tabular}


the size $58 \times 58$ for $A$. The values of $c_{r}$ and $c_{i}$ shown in this table have converged quite well. This can be seen by an examination of Table 1, in which $c_{r}$ and e-folding time are presented as a function of the size of the matrix A. The bars in table 2 represent computations which gave stable roots only.

A rather curious feature of the results presented in Table 2 is that when instability occurs, we seem to obtain two pairs of complex roots $c=c_{r} \pm i c_{i}$ and $c^{-}=c_{r}^{\prime} \pm i c_{i}^{\prime}$ and they are located symmetrically about the mean speed $\frac{1}{2}\left(\bar{u}_{1}+\bar{u}_{2}\right)$ as far as the phase speeds are concerned. This situation holds for $\bar{u}_{2}<45 \mathrm{~m} \cdot \mathrm{sec}^{-1}$. Then we obtain a small stable region before passing on into another region of instability around $\bar{u}_{2}=47.5 \mathrm{~m} \cdot \mathrm{sec}^{-}$. Further, the instability seems to be bounded on the long-wave and short-wave side of the spectrum.

The symmetry of the $c_{r}$ 's of the two pairs of roots with respect to the mean speed-as well as the occurrence of two pairs of complex rootsis rather an accident and is due to the choice $\bar{D}_{1}=\bar{D}_{2}$. If $\bar{D}_{1} \neq \bar{D}_{2}$ this symmetry is no longer realized as will be shown later and one of the two sets of complex roots may sometimes disappear as a consequence. The implications of the existence of two unstable roots when they occur together are important in the considerations of the representation of an arbitrary disturbance. In the conventional stability analysis, the existence of an unstable normal mode implies that any arbitrary disturbance will start growing at a rate essentially determined by this mode. However, if two unstable normal modes are present with approximately the same growth rates, whether an arbitrary disturbance starts to grow initially--and if so, at what rate--depends very much on the phase of these two normal modes relative to one another. 
Table 2 Spectral model, $N=9, W=500 \mathrm{~km} ., \varepsilon=.98, \bar{D}_{1}=\bar{D}_{2}=5 \mathrm{~km} ., \bar{u}_{1}=10 \mathrm{~m} \cdot \mathrm{se}$.

Values of $c_{r}$ in $\mathrm{m} / \mathrm{sec}$ and e-folding time in days (in parentheses) are as a function of $\left(\bar{u}_{2}-\bar{u}_{l}\right)$ and wave number.

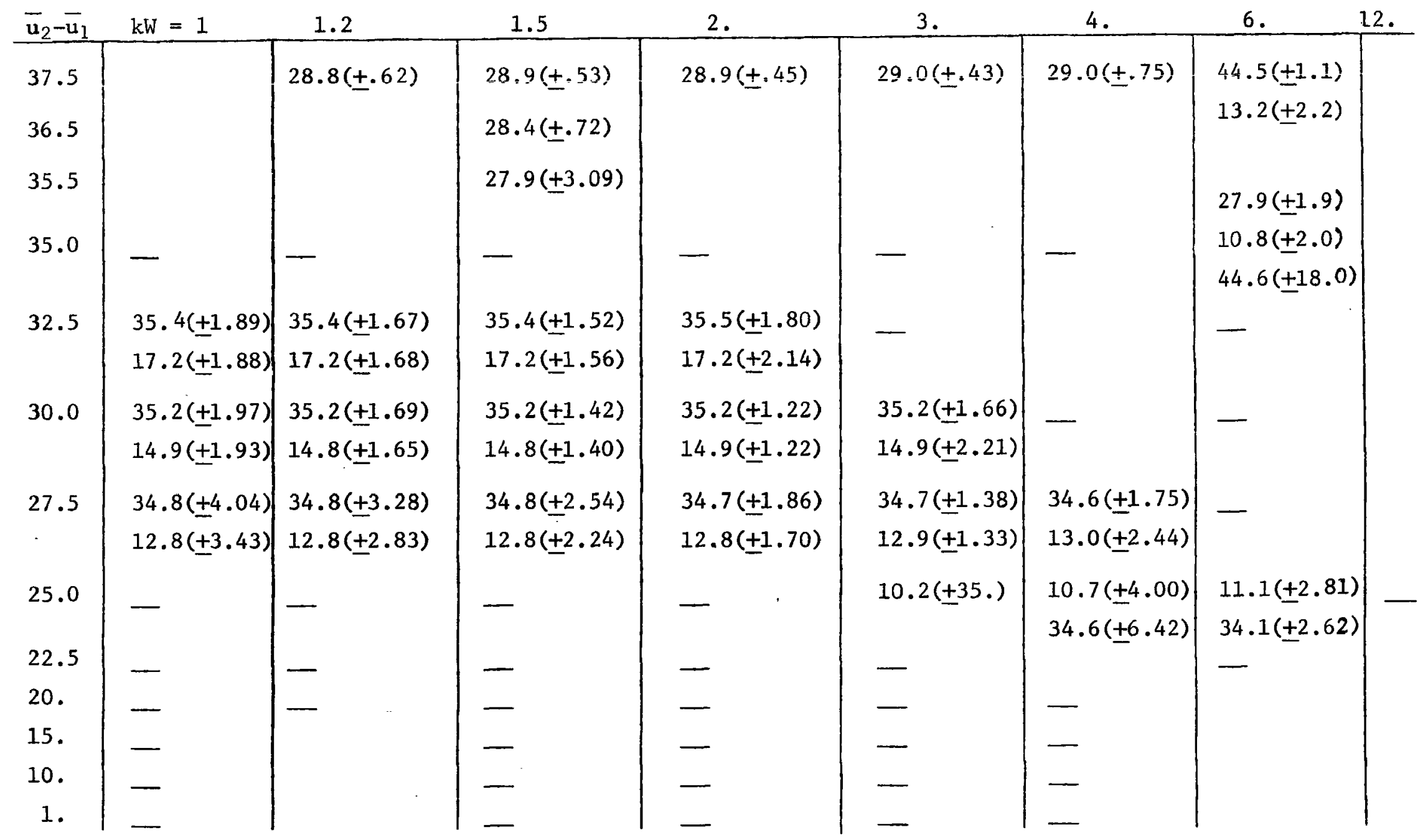


Let us now examine the mechanism that is responsible for producing the instability. Information on the mechanism can be obtained by tracing the change in the value of the roots as $\bar{u}_{2}$ is increased. As mentioned earlier, there are basically three types of modes in the mode1.

(I) external gravitational modes

(II) internal gravitational modes

(III) rotational modes

When the secular determinant $\mathbb{A}$ is truncated at a size $M \times M$, we get $M$ roots which can then be assigned into each one of the above categories. Tracing the changes in the values of these roots as the shear is increased--or what is the same, $\bar{u}_{2}$ is increased--helps us identify the mechanism initiating the instability. To keep the matters simple, instead of tracing the changes in all the $M$ roots, we will restrict our attention to only those roots which eventually cause instability when the shear has become sufficiently high. It turns out that these roots are partly rotational and partly gravitational (interal). The nature of these roots can be ascertained by comparison with exact solutions for simple cases. Tables 3 shows these potentially unstable roots as a function of $\overline{\mathrm{u}}_{2}$ for a specific wave number $\mathrm{kW}=1.5$. In this table, we have also included for comparison the quasi-geostrophic rotational modes, and the internal gravitational modes for $f=0$ ("filtered solutions"). These results show that each pair of roots is symmetrically located with respect to the mean speed $\bar{U}=\frac{1}{2}\left(\bar{u}_{1}+\bar{u}_{2}\right)$. We notice from Table 3 that the $c_{r}$ 's for the rotational modes are in the range $\overline{\mathrm{u}}_{1}<\mathrm{c}_{\mathrm{r}}<\overline{\mathrm{u}}_{2}$ and the $c_{\mathrm{r}}$ 's for the gravitational modes are in the range $c_{r}<\bar{u}_{1}$ and $c_{r}>\bar{u}_{2}$ when the shear is small. As the shear- 
Table 3: The Mechanism of mixed-mode instability

$\mathrm{k}=1.5 / 500 \mathrm{~km} ., \mathrm{W}=500 \mathrm{~km}, \varepsilon=.98, \overline{\mathrm{D}}_{1}=\overline{\mathrm{D}}_{2}=5 \mathrm{~km}, \overline{\mathrm{u}}_{1}=10 \mathrm{~m} / \mathrm{sec}$, upper table : $\quad c_{1}$ and $c_{2}$ quasi-geostrophic rotational modes. $c_{3}$ and $c_{4}$ basic internal gravity modes for $f=0$. Lower table: Basic rotational and internal gravitational modes of the spectral solution. e-folding time in parentheses. $\bar{U}=\frac{1}{2}\left(\bar{u}_{1}+\bar{u}_{2}\right)$

\begin{tabular}{|c|c|c|c|c|}
\hline $\bar{u}_{2}$ & $c_{3}-\bar{U}$ & $c_{1}-\bar{U}$ & $c_{2}-\bar{U}$ & $c_{4}-\bar{U}$ \\
\hline 30 & -19.8 & -6.5 & 6.4 & 19.8 \\
\hline 32.5 & -19.0 & -7.3 & 7.2 & 19.0 \\
\hline 35 & -18.3 & -8.1 & 8.0 & 18.3 \\
\hline 37.5 & -17.4 & -8.9 & 8.8 & 17.4 \\
\hline 40 & -16.3 & -9.7 & 9.6 & 16.3 \\
\hline 42.5 & -15.0 & -10.5 & 10.4 & 15.0 \\
\hline 43.5 & -14.4 & -10.8 & 10.7 & 14.4 \\
\hline 44.5 & -13.9 & -11.1 & 11.0 & 13.9 \\
\hline 45 & -13.6 & -11.3 & $11: 2$ & 13.6 \\
\hline 45.5 & -13.2 & -11.5 & 11.4 & 13.2 \\
\hline 46.5 & -12.5 & -11.8 & 11.7 & 12.5 \\
\hline 47.5 & -11.8 & -12.1 & 12.0 & 11.8 \\
\hline 30 & -17.2 & -6.8 & 6.7 & 17.3 \\
\hline 32.5 & -15.4 & -7.9 & 7.8 & 15.5 \\
\hline 35 & -13.6 & -9.1 & 8.9 & 13.8 \\
\hline 37.5 & $-11.0 \pm 1.72 i$ & $(2.24)$ & \multicolumn{2}{|c|}{$11.0+1.52 i$} \\
\hline 40. & $-10.2 \pm 2.76 i$ & $(1.40)$ & \multicolumn{2}{|c|}{$10.2+2.72 i \quad(1.42)$} \\
\hline 42.5 & $-9.1 \pm 2.47 i$ & $(1.56)$ & \multicolumn{2}{|c|}{$9.1 \pm 2.54 i$} \\
\hline 43.5 & $-8.5 \pm 1.57 i$ & $(2.46)$ & \multicolumn{2}{|c|}{$8.5 \pm 1.74 i \quad(2.21)$} \\
\hline 44.5 & -9.9 & -5.5 & 5.7 & 9.8 \\
\hline 45. & -10.4 & -3.6 & 3.8 & 10.3 \\
\hline 45.5 & -10.9 & \multicolumn{2}{|c|}{$.1 \pm 1.25 i(3.09)$} & 10.8 \\
\hline 46.5 & -11.6 & \multicolumn{2}{|c|}{$.1 \pm 5.36 i(.72)$} & 11.5 \\
\hline 47.5 & -12.2 & \multicolumn{2}{|c|}{$.1 \pm 7.33 i(.53)$} & 12.2 \\
\hline
\end{tabular}




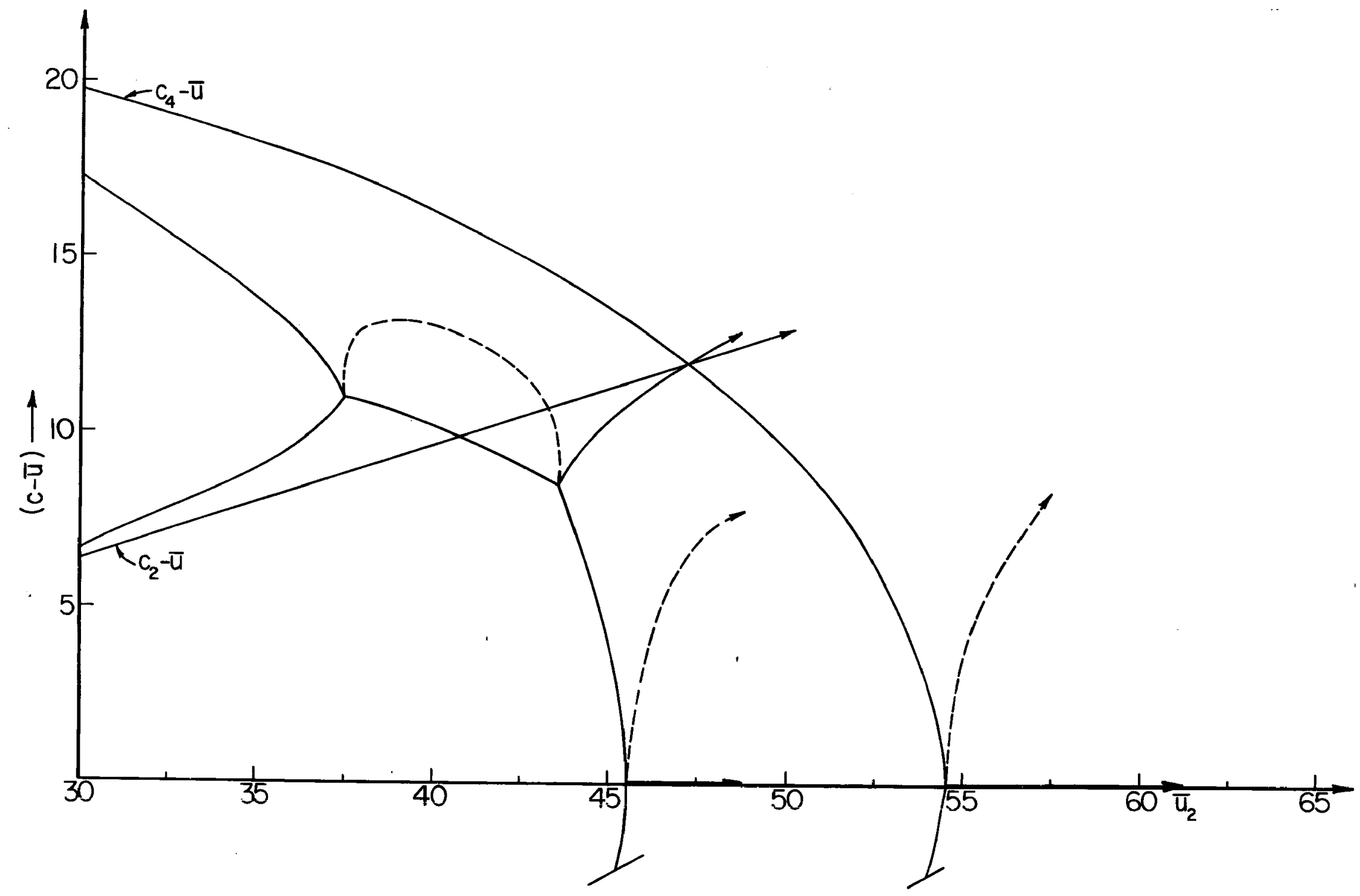

Figure 2. Illustration of the confluence of gravitational and rotational modes to initiate instability. Curves labelled $c_{4}-\bar{u}$ and $c_{2}-\bar{u}$ are the filtered gravitational and rotational modes. The unlabelled curves correspond to the non-filtered situation. The dashed curves represent $c_{i}$, whose magnitude is given by the vertical distance between the solid line and the dashed line. 
-or $\bar{u}_{2}$-increases one member of the internal gravitational mode pair approaches one of the members of the rotational mode pair while the other member of the gravitational mode pair approaches the second member of the rotational mode pair. This process is illustrated in Figure 2. In this figure, the dash-dot-line shows the behaviour of the "filtered" solution labelled $c_{2}$ and $c_{4}$ in Table 3 - that is, the pure internal gravity wave solution with $f=0$ and the quasi-geostrophic rotational wave-individually as a function of $\bar{u}_{2}$. The other two filtered roots $c_{1}, c_{3}$ are in the lower half of the diagram forming a mirror reflection and consequently are not shown in Figure 2. When filtering is not used and the rotational and gravitational modes exist together, the behaviour of the roots is shown by the solid lines. It is seen that the modified roots $c_{2}$ and $c_{4}$ come together at a critical value of $\bar{u}_{2}$ and instability is then initiated. The magnitude of $c_{i}$ is then given by the vertical distance between the solid and dashed lines. The instability persists until another critical value of $\overline{\mathbf{u}}_{2}$ is reached and represents the primary region of instability shown in Table 2. At the second critical value of $\bar{u}_{2}$ the modes separate and we get a stable region. By a comparison with the filtered solutions, we see that for further increase in $\bar{u}_{2}$ the value of $c-\bar{U}$ decreases for the gravitational mode $c_{4}$ and increases for the rotational mode $c_{2}$. This situation continues until another critical value of $\bar{u}_{2}$ is reached when the gravitational mode $c_{4}$ meets its counter part $c_{3}$ coming from below the axis $c-\bar{U}=0$. At this point, the instability occurring for higher values of $\bar{u}_{2}$ in Table 2 is obtained. The instability now is generated by gravitational modes alone and hence is of the KelvinHelmholtz type modified by effects of rotation. The Kelvin-Helmholtz 
instability in the presence of rotation occurs for a lower shear (or $\bar{u}_{2}$ ) than in the case of $f=0$, as shown in Figure 3 where the filtered gravitational modes $c_{3}, c_{4}$ come together at a much larger value of $\overline{\mathrm{u}}_{2}$ to generate instability. Thus rotation, in addition to introducing the primary instability region, also seems to increase the KelvinHelmholtz instability. In any case, the primary instability is the result of an interaction between a wave of the internal gravitational type and a wave of the rotational type. The choice $\bar{D}_{1}=\bar{D}_{2}$ in the present example produces the result that when $c_{2}$ and $c_{4}$ merge together, so do the roots $c_{1}$ and $c_{3}$ yielding the second complex mode. When $\bar{D}_{1} \neq \bar{D}_{2}$, one of the two sets of complex modes may be eliminated. This is due to the fact that the $c_{r}$ 's of the gravitational and rotational modes are no longer symmetric with respect to the mean speed and when a confluence of the above type occurs between say $c_{2}$ and $c_{4}$ the other members $c_{1}$ and $c_{3}$ are still far apart. This will be discussed in more detail in connection with Table 4.

The instability mechanism generated by an interaction between a gravity mode and a rotational mode is a rather unique one. This mechanism straddles the fence--so to say--between the instability mechanisms for the large scale motions and the small scale motion. In the case of the large scale motions, one can study the dynamics of instability in the framework of a filtered system of equations in which the gravity waves are no longer present. Hence the instability, when obtained, must be brought about purely by the rotational modes. On the other hand, earth's rotation is relatively unimportant for small scale motions and their instability, like the Helmholtz instability for example, is purely governed by the spectrum of the gravity modes. 
For medium scale motions, as the ones considered here, the spectrum of the gravitational and rotational modes are equally important and elimination of either one of these classes of modes would eliminate any possible instability that the system is capable of exhibiting. Even though in the model considered so far, the free surface is supposed to represent the tropopause, in reality the atmosphere extends far above the tropopause. To simulate this, we have superimposed a third layer of infinite depth on top of the second layer. In order to keep the slope of the second surface unchanged, the third layer is assumed to have the same constant translational speed as the second layer under equilibrium conditions; that is, $\bar{u}_{3}=\bar{u}_{2}$. The dynamical equations formally look the same as those given by (2.8-10) except for a slight re-definition of the pressure gradient terms and the method of analysis of the preceding section goes through without any modifications. The results for $c_{r}$ and the growth rate for the case with infinitely deep third layer are shown in Table 4 as a function of $\varepsilon^{\prime}=\rho_{3} / \rho_{2}$ for a wave number $\mathrm{kW}=1.5$ and $\vec{u}_{2}=40 \mathrm{~m} / \mathrm{sec}$. It is seen from this that when $\varepsilon^{\prime}$ is less than about 0.9 , there does not appear to be much effect of the third layer on the stability character. However, when $\varepsilon^{\prime}=0.98$, one of the two unstable pairs has disappeared and we now have only one unstable normal mode. This mode propagates with a phase speed closer to the speed of the upper layer. The mechanism of instability may be shown to be still the same as before. The presence of the upper layer has a slight tendency to reduce the stability.

Continuing with the effects of upper layers on the stability, let us now consider the effect of increasing the depth of the upper layer 
Table 4. Effect of increasing depth of second layer or adding a third layer of infinite depth. $\bar{u}_{3}=\bar{u}_{2}, \varepsilon^{\prime} \equiv \rho_{3} / \rho_{2}$. Values of the parameters $\mathrm{k}=1.5 / 500 \mathrm{~km}, \mathrm{~W}=500 \mathrm{~km}, \varepsilon=.98, \overline{\mathrm{u}}_{1}=10 \mathrm{~m} / \mathrm{sec}, \overline{\mathrm{u}}_{2}=$ $40 \mathrm{~m} / \mathrm{sec}, \bar{D}_{1}=5 \mathrm{~km}$

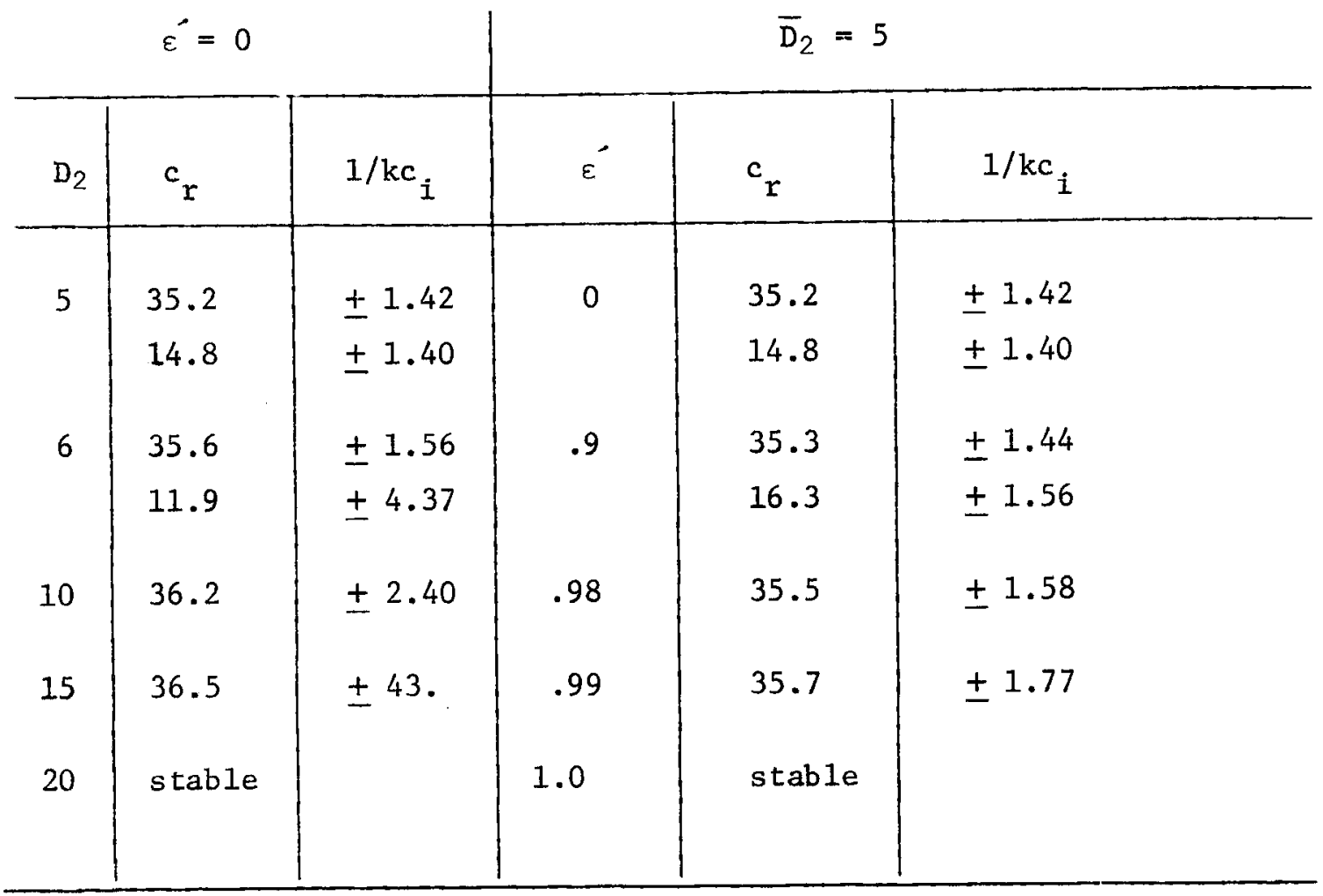



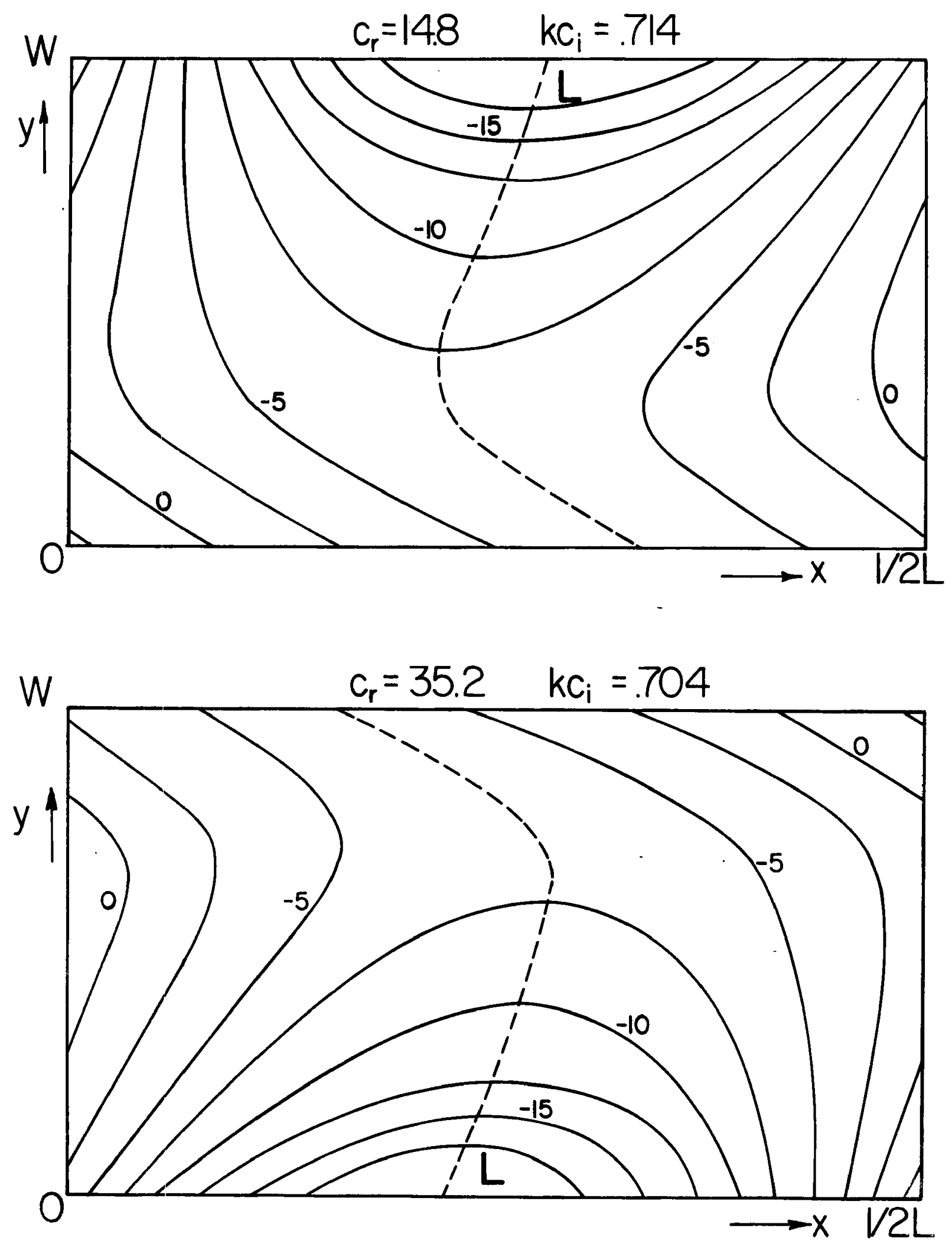

Figure 3. Structures of the two unstable modes in terms of $h_{1}$ in the prinary instability region. 


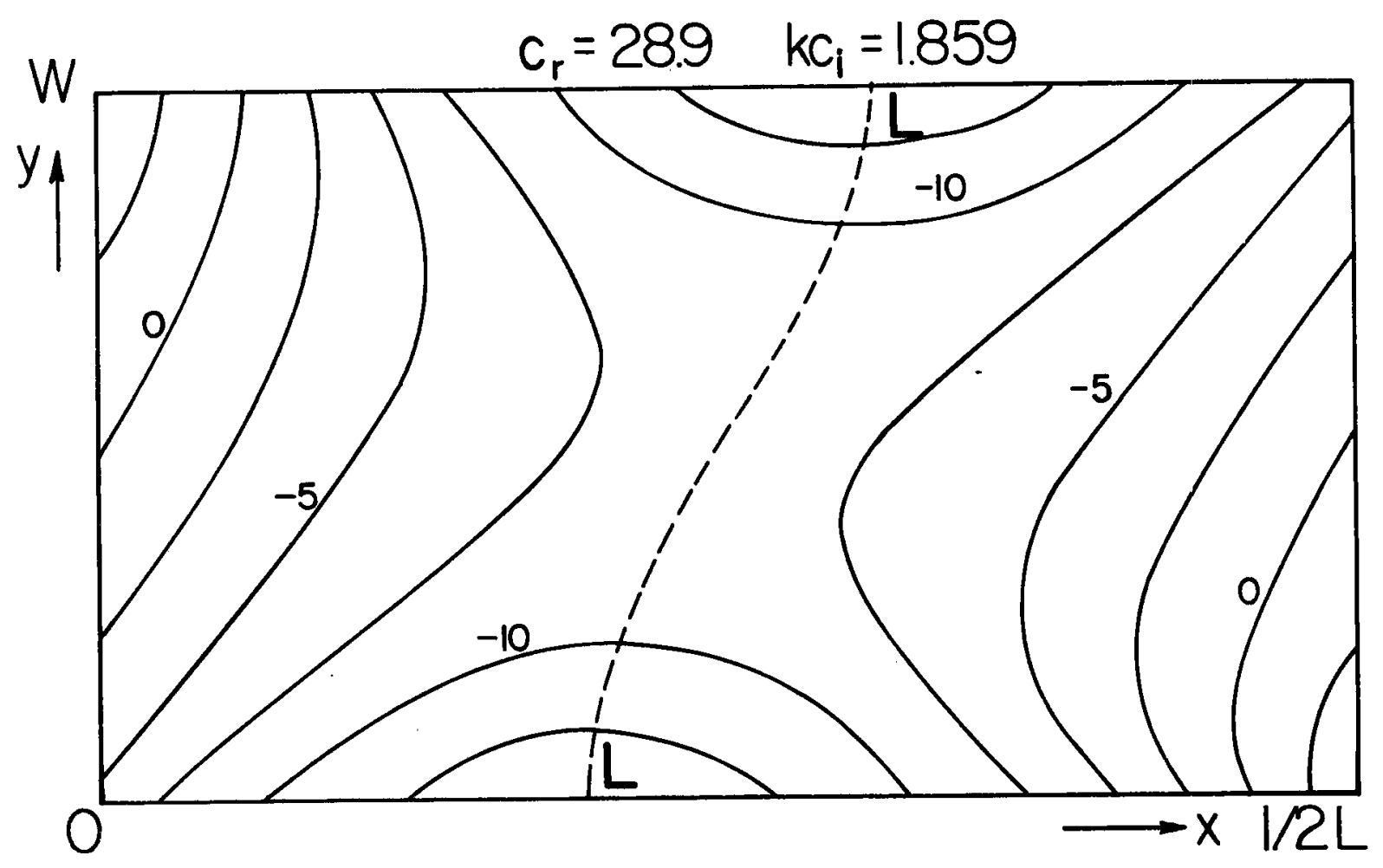

Figure 4. Structure of the unstable mode in the secondary region of instability. 
while keeping the lower layer depth the same as before. The results of this are also given in Table 4. As one would expect from elementary reasoning, the increase in the depth of the upper layer tends to stabilize the configuration. It may also be noticed that once the uniform depths $\bar{D}_{1}$ and $\bar{D}_{2}$ are quite different, one of the unstable modes is eliminated.

We next consider the effect of different depths on the stability. Table 5 shows these results. It is clear from this table that instability is initiated at a smaller value of the shear $\left(\bar{u}_{2}-\right.$ $\bar{u}_{1}$ ) if $\bar{D}_{1}$ is small. Further, the region of stability that is obtained in Table 2 for higher values of shear is decreased for $\bar{D}_{1}=4 \mathrm{~km}$ and altogether disappeares for $\bar{D}_{1}=3 \mathrm{~km}$. As before, the presence of a third layer of different density or making $\bar{D}_{1} / \bar{D}_{2} \neq 1$, eliminates one of the two unstable pairs.

The structures of the unstable modes are shown in Figures 3 and 4. The top picture in Figure 3 corresponds to the mode which is eliminated by having either a third layer or an unequal $\bar{D}_{1}$ and $\bar{D}_{2}$. The bottom picture in Figure 3 shows the height distribution for the mode with its phase speed closer to the upper layer speed. It shows that development takes place closer to the $y=0$ boundary or the low levels. Figure 4 shows the structure of the unstable normal mode at $\bar{u}_{2}=47.5 \mathrm{~m} \cdot \mathrm{sec}^{-1}$.

5. Other cases of mixed-mode instability.

In this section we will compare the two-fluid system with lateral boundaries with simpler systems for which exact solutions exist. For that purpose we write first the linearized equations (3.2)(3.4) in terms of a stream-function and flow potential $(\backslash V=\nabla X-[\nabla \psi])$ 
Table 5. Effect of different uniform depths for the case of $\mathrm{kW}=1.5$, $\bar{u}_{1}=10 \mathrm{~m} / \mathrm{sec}, \varepsilon=0.98$. Given in the table are $c_{r}$ and $1 / \mathrm{kc}_{i}$.

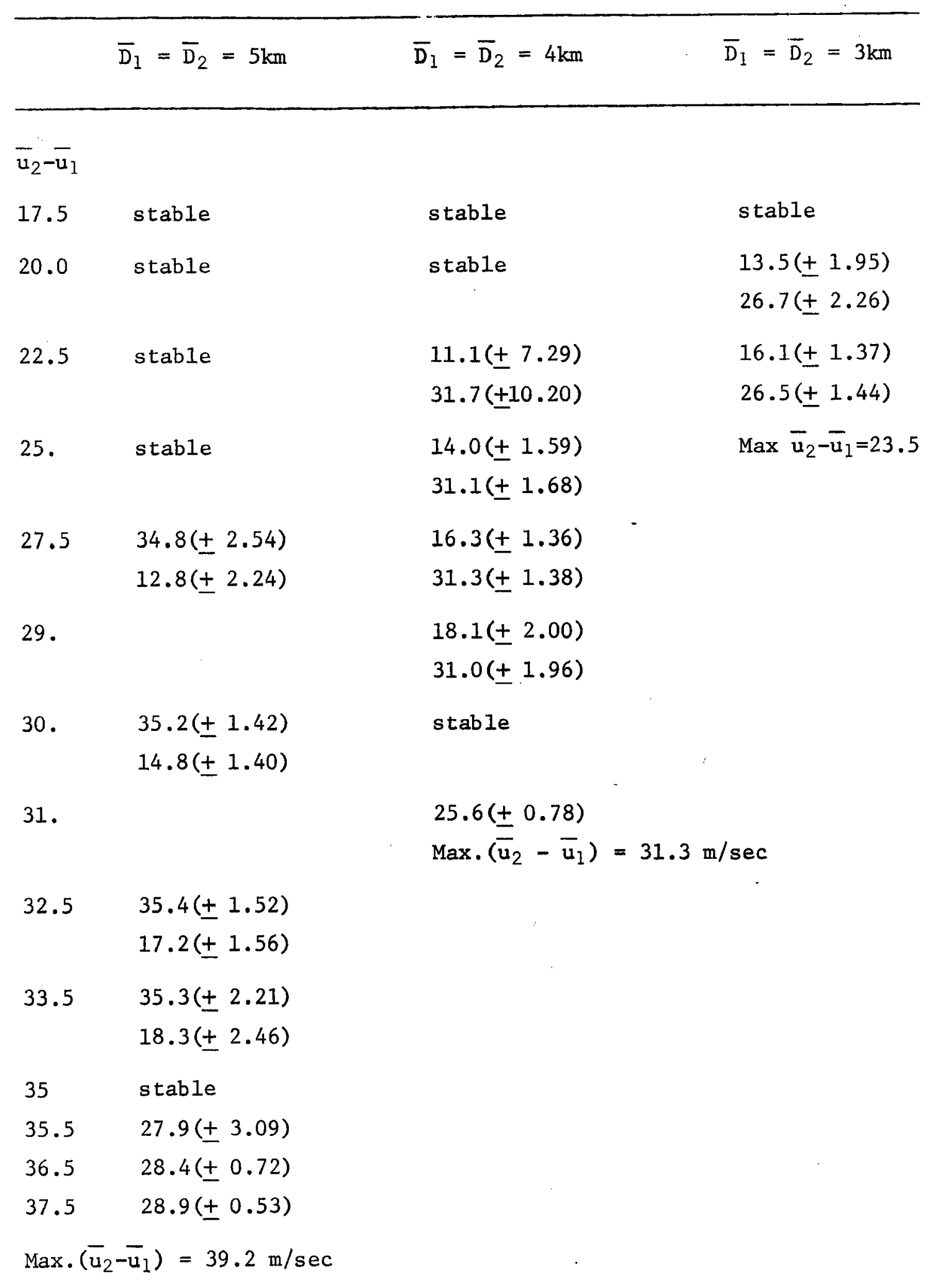


and form the divergence and vorticity equations

$$
\begin{aligned}
& \left(\frac{\partial}{\partial t}+i k \bar{u}_{j}\right) \nabla^{2} x_{j}-f \nabla^{2} \Psi_{j}+g \nabla^{2} H_{l}+g \gamma \nabla_{j}^{2} H_{2}=0 \\
& \left(\frac{\partial}{\partial t}+i k \vec{u}_{j}\right) \nabla^{2} \Psi_{j}+f \nabla^{2} x_{j}=0 \\
& \left(\frac{\partial}{\partial t}+i k \vec{u}_{j}\right) H_{j}+i k \bar{\alpha}_{j} f \Psi_{j}+\bar{h}_{j} \nabla^{2} x_{j}+\bar{\alpha}_{j} f \frac{\partial x_{j}}{\partial y}=0
\end{aligned}
$$

where we have assumed periodicity in $\mathrm{x}$ as in (3.1) and therefore $\Psi_{j}=\Psi_{j}(y, t), x_{j}=x_{j}(y, t)$, and $\nabla^{2}=-k^{2}+\partial^{2} / \partial y^{2}$.

Let us first consider the simplified system where $\bar{h}=\bar{D}=$ constant and the non-geostrophic advection (the last term) in the last equation is negligible.

$\left(\frac{\partial}{\partial t}+i k \bar{u}_{j}\right) H_{j}+i k \bar{\alpha}_{j} f \Psi+\bar{D}_{j} \nabla^{2} x_{j}=0$

The system allows for trigonometric or periodic solutions in $y$, such that the operator $\nabla^{2}$ becomes simply $-\left(k^{2}+\ell^{2}\right)$ where $l$ is the $y$-wave number. Except for the first term of (5.1) the equations are those of the familiar quasi-geostrophic system. However, the equations allow for internal and external gravitational modes in addition to the rotational modes, and therefore we have here a possibility for the mixed mode instability to occur. Indeed this type of instability is found at the short end of the wavelength spectrum (Simons and Rao, 1970). If the gravity modes are filtered out, eg., by the quasi-geostrophic approximation, then short waves are always stable--the equivalent of the short wave cut off in the Eady (1949) model. This cut-off takes place at wavelengths of the order of $1000-2000 \mathrm{~km}$, depending on the $y$-wave number $\ell$. On the other hand, if the rotational modes are filtered out by setting $f=0$ we can only have the Kelvin-Helmholtz type instability which occurs only at very short wavelengths, at least 
for the shears here considered. The non filtered system (5.1), (5.2), and $(5.3 \mathrm{~b})$, however, often produces two pairs of complex roots in the region between the Helmholtz-and the geostrophic instability. The wave speed of the first pair is close to the mean zonal velocity of the lower layer and the second pair moves with nearly the upper layer mean speed. Their growth rates tend to be almost equal, and the e-folding times are in many cases less than one day. The mixed mode instabilities occur in patches in a shear-wavelength diagram and disappear if we go to higher or lower wave number or higher or lower shear. The $y$-wave number has only a slight influence on these short waves (below $1000 \mathrm{~km}$ ) but the required shear is of the order of $30 \mathrm{~m} / \mathrm{sec}$ which can only be reached for channel widths less than about $650 \mathrm{~km}$ if the interface is not to touch the ground or the free surface (assuming $\overline{\mathrm{D}}_{1}=\overline{\mathrm{D}}_{2}=5 \mathrm{~km}$.)

It has been suggested in the meteorological literature (Phillips, 1964) that the non-geostrophic effect represented by the rate of change of divergence in (5.1) may not be the most important one, and that $y$-dependent terms such as the last term of (5.3) should also be retained. Although the simplified system (5.1), (5.2), (5.3b), does indeed produce the above discussed mixed-mode instability for the geostrophically-stable shorter wavelengths, the system seems to allow for only one pair of unstable roots for longer wave lengths, and this instability is quite similar to that obtained from the geostrophic

* It turns out that the Kelvin-Helmholtz instability for a two-fluid system with a free surface is quite different from what one obtains in the case of the classical two-infinite fluid system or in the case of a two fluid system bounded at top and bottom by rigid boundaries. This is described elsewhere (Simons and Rao 1970). 
equations. We have therefore investigated the effects of the nongeostrophic terms in (5.3) in some more detail, in particular at the short wave end of the quasi-geostrophic instability region.

Choosing as before $\varepsilon=.98, \bar{D}_{1}=\bar{D}_{2}=5 \mathrm{~km}$, we find that quasigeostrophic instability can only occur for $\sqrt{k^{2}+e^{2}}<5 \times 10^{-6} 0^{-1}$ Considering now a wavelength of $2100 \mathrm{~km}(\mathrm{k}=1.5 / 500 \mathrm{~km})$ we see that this wave is geostrophically stable in the $500 \mathrm{~km}$ channel (as are all wavelengths) while the wave becomes just unstable in a 1000-km channel and is well in the unstable part of the spectrum for a $2000 \mathrm{~km}$ channel. The condition that the interface should not touch the ground nor the free surface requires that the shear be less than $3920 \overline{\mathrm{D}} / \mathrm{W} \mathrm{m} \cdot \mathrm{sec}^{-1}$. Assuming again $\bar{u}_{1}=10 \mathrm{~m} \cdot \mathrm{sec}^{-1}$ we have the conditions $\bar{u}_{2}<49.2 \mathrm{~m} \cdot \mathrm{sec}^{-1}$ for $W=500 \mathrm{~km}, \bar{u}_{2}<29.6 \mathrm{~m} \cdot \mathrm{sec}^{-1}$ for $W=1000 \mathrm{~km}$ and $\bar{u}_{2}<19.8 \mathrm{~m} \cdot \mathrm{sec}^{-1}$ for $W=2000 \mathrm{~km}$. The first column of table 6 shows the quasi-geostrophic instabilities for these channel widths and for a few vertical shears. The second column shows the solution of the simplified system with (5.3) replaced by (5.3b). No mixed-mode instability occurs for these values of the basic parameters.

At first glance it would seem as if the approximation (5.3b) is not necessary to obtain an exact solution. If we only treat $\bar{h}$ as a constant in (5.3), the system allows for an exact periodic solution in $\mathrm{y}$. In the meteorological literature a similar approach has been followed in solving the non-geostrophic Eady problem. It follows immediately from the character of the coefficient matrix that such an approach can only produce complex wave speeds. Actual computations show that generally the external gravity modes (with wave speeds over $300 \mathrm{~m} / \mathrm{sec}$ ) show the largest growth rates. From physical considerations 
Table 6 Comparison of channel solutions with exact solutions

$\mathrm{L}=2095 \mathrm{~km}(\mathrm{k}=1.5 / 500 \mathrm{~km}), \varepsilon=.98, \overline{\mathrm{D}}_{1}=\overline{\mathrm{D}}_{2}=5 \mathrm{~km}, \overline{\mathrm{u}}_{1}=10 \mathrm{~m} / \mathrm{sec}$.

A: exact quasi-geostrophic solution for the channel

B: exact solution of system (5.1), (5.2), and (5.3b) periodic in $y$, for $\ell=\frac{\pi}{W}$

C: Channel solution for system (5.1-3) for $\bar{h}=D=$ constant

D: Channel solution for system (5.1-3) (C and D are spectral solutions)

$c_{r}$ given without parentheses and e-folding time $i / k c_{i}$ given within parentheses.

\begin{tabular}{|c|c|c|c|c|c|}
\hline W & $\overline{u_{2}}$ & A & B & $\mathrm{C}$ & $\mathrm{D}$ \\
\hline $500 \mathrm{~km}$ & $\begin{array}{l}40.0 \\
49.0\end{array}$ & stable & $\begin{array}{l}\text { stable } \\
\text { stable }\end{array}$ & $\begin{array}{l}\text { stable } \\
16.8( \pm .87) \\
42.1( \pm .87)\end{array}$ & $\begin{array}{ll}14.8 & ( \pm 1.40) \\
35.2 & ( \pm 1.42) \\
29.6( \pm .41)\end{array}$ \\
\hline $1000 \mathrm{~km}$ & $\mid \begin{array}{l}20.0 \\
29.0\end{array}$ & $\begin{array}{l}14.9( \pm 4.74) \\
19.4( \pm 2.41)\end{array}$ & $\begin{array}{l}14.9( \pm 4.70) \\
19.4( \pm 2.32)\end{array}$ & $\begin{array}{l}14.9(+6.85) \\
\text { stable }\end{array}$ & $\begin{array}{l}14.9( \pm 4.69) \\
13.9( \pm 2.19) \\
25.7( \pm 2.59)\end{array}$ \\
\hline $2000 \mathrm{~km}$ & 19.5 & $\begin{array}{l}12.4( \pm 2.98) \\
12.4( \pm 10.58)^{*} \\
14.6( \pm 1.56) \\
14.7( \pm 4.53)^{*}\end{array}$ & $\begin{array}{ll}12.4 & ( \pm 2.97) \\
12.4 & ( \pm 10.53) \\
14.6 & ( \pm 1.54) \\
14.7 & ( \pm 4.98)\end{array}$ & $\begin{array}{l}12.4( \pm 11.66) \\
12.4( \pm 2.97) \\
14.7( \pm 7.05) \\
14.6( \pm 1.54)\end{array}$ & $\begin{array}{l}12.4( \pm 6.37) \\
12.4( \pm 3.20) \\
15.5( \pm 2.27) \\
13.6( \pm 2.37)\end{array}$ \\
\hline
\end{tabular}

* exact solutions for second harmonic, ie., for $\mathrm{W}=1000 \mathrm{~km}$ 
we may discard this instability as spurious. We have, therefore, solved the particular system (5.1-3) with $\overline{\mathrm{h}}=\overline{\mathrm{D}}=$ constant for the channel, using the spectral technique. The results are shown in column $C$ of table 6 . Comparison with column B indicates that the last term of (5.3) could be important for higher shears and small channel widths. The two pairs of unstable roots for $\mathrm{W}=2000 \mathrm{~km}$ simply reflect the fact that the spectral solution also includes the geostrophically unstable wave corresponding to $\mathrm{W}=1000 \mathrm{~km}$.

The last column of table 6 presents the spectral solution of the complete system (5.1-3) which is equivalent to system (3.2-5). It is seen that the mixed-mode instability slowly changes to the quasigeostrophic instability as the channel width increases. Nevertheless another type of mixing seem to occur between the two pairs of unstable geostrophic modes for $W=2000, \bar{u}_{2}=19.5 \mathrm{m.sec} .^{-1}$ This is suggested by comparison of these roots with the corresponding ones in column $\mathrm{C}$. It can be verified that no gravitational modes are involved here, since the gravity waves can be traced to be stable. In general comparing the two last columns of table 6 , which only differ in that $\bar{h}$ is treated as a constant or a function of $y$, we must conclude that the variation of $\bar{h}$ in (5.3) tends to favor the occurrence of mixed mode instability.

\section{Summary and Conclusions}

The problem of non-geostrophic instability of a two-fluid system in a channel has been considered. It was shown that the primary instability in this model is realised as a result of an interaction between a gravitational and rotational mode. It is possible to obtain 


\section{References}

Arnason, G., 1963: The stability of nongeostrophic perturbations in a baroclinic zonal flow. Tellus, 15, 205-229.

Charney, J. G. 1947: The dynamics of long waves in a baroclinic Westerly current. Journal of Meteorology, 4, 135-162.

Derome, J. F. and A. Wiin-Nielsen 1966: On the baroclinic stability of zonal flow in simple model atmospheres. Technical Report. College of Engineering, Univ of Michigan, Ann Arbor, Mich, 93pp.

Eady, E. 1949: Long waves and cyclone waves. Tellus, 1, 33-52. Kotschin, N. 1932: Ueber die Stabilität von Margulesschen diskontinuitäts Feächen. Beiträge zur Physik der freien Atmosphäre, 18, 129-164.

Orlanki, I. 1968: Instability of frontal waves. Journal of the Atmospheric Sciences, 25, 178-200.

Phillips, N. A. 1964: An overlooked aspect of the baroclinic stab :y problem. Tellus, 16, 268-270.

Sela, J. and S. J. Jacobs 1968: On baroclinic and barotropic ageostrophic stability. Technical Report. College of Engineering, Univ. of Michigan, Ann Arbor, Michigan, 98pp.

Simons, T. J. and D. B. Rao 1970: Instability of rotational and gravitational modes of oscillation. (To be published).

Solberg, H. 1928: Integrationen der atmosphärischen Störungsgleichungen. Geofysiske Publikasjoner, 5, No. 9, $120 \mathrm{pp}$.

Stone, P. 1966: On non-geostrophic baroclinic stability. Journal of Atmospheric Sciences, 23, 390-400. 